\title{
A dynamic battery model for co-design in cyber-physical systems
}

\author{
Fumin Zhang, Zhenwu Shi \\ School of Electrical and \\ Computer Engineering \\ Georgia Inst. of Technology \\ Savannah, Georgia 31407 \\ Email: fumin, zwshi@gatech.edu
}

\author{
Wayne Wolf \\ School of Electrical and \\ Computer Engineering \\ Georgia Institute of Technology \\ Atlanta, Georgia 30332 \\ Email:wolf@ece.gatech.edu
}

\begin{abstract}
We introduce a dynamic battery model that describes the variations of the capacity of a battery under time varying discharge current. This model supports a co-design approach for cyber-physical systems where control laws, online scheduling algorithms and battery management can be designed jointly to balance performance of the plant, the computing device, and the batteries. The input-output relationship of our model is equivalent to the RakhmatovVrudhula-Wallach (RVW) model that has been verified by high fidelity simulations and experiments. The major improvement of our model is that it allows online prediction of battery life. This improvement is significant since for cyber-physical systems, control laws and online scheduling algorithms draw current from batteries according to the state of the plant and the processor, which cannot be determined at the design stage. Using our model, the optimal discharge profile for a square wave current are determined and simulation results are provided to compare battery life for different discharge profiles.
\end{abstract}

\section{Introduction}

A key idea for Cyber-physical systems research is to codesign different controlling mechanisms to balance performance in both physical systems and computing systems [17], [14], [13], [9], [1]. An important class of applications are mobile sensor networks where each sensor node is also a mobile robot that is able to reconfigure the structure of the network [4], [16], [15]. Due to the requirement for mobility, most mobile sensor networks are powered by batteries. Until now, the majority of batteries used are electro-chemical batteries. It has been understood that such batteries are complex chemical/physical systems by themselves and possess interesting "nonlinear" discharge behaviors. However, classical approaches in cyber-physical co-design tend to ignore these battery bahaviors. For example, in classical optimal control, controller design is often based on minimizing a cost function that includes the averaged power of control effort, and the battery is idealized as a constant voltage source providing the desired power. For a real battery, such minimized averaged power does not translate to increased battery life directly.

According to battery and VLSI design literature e.g. [3], [12], the discharge current is supported by the change of concentration of electrolytes near the anode or the cathode of a battery. The chemical processes of oxidation and reduction reduce the concentration of electrolytes near the electrodes and create a spatial gradient of concentration within the battery. Driven by the concentration gradient, electrolytes diffuse to the electrodes from other areas of the battery. When the concentration at the electrodes drops below a certain threshold, a battery fails to support discharge current, causing failure to devices it supports. At this moment, the discharge cycle has to stop and the battery needs to be recharged or replaced. Note that there may be a significant amount of active electrolytes left in the battery when a discharge cycle ends. Therefore, the actual capacity of a battery is significantly lower than the theoretical capacity that is the total amount of electrolytes contained in a battery. When the battery is discharged under a pulsed discharge current, during the idle time when current is interrupted, the diffusion process increases electrolyte concentration at the electrodes. This produces the recovery effect that makes the battery appears to have regained portions of its capacity. The amount of capacity recovered depends on the amount of active electrolyte available. With properly chosen idle time, the recovery effect significantly improves battery performance in two aspects. First, the battery is able to deliver current impulses that several times larger than a constant current. This is because the diffusion process allows charges to redistribute after a quick discharge is drawn. Second, if the averaged currents are identical, impulsive current increases the total charge delivered i.e. the actual capacity and lifetime of the battery is increased.

Battery modeling aims to simulate these behaviors by computational models [12]. Existing models are typically categorized as physical, empirical and abstract models. Physical models are based on the complex electrochemical process within a battery [5], hence requiring long time to compute. Empirical models uses very simple equations to 
describe specific battery behavior [8], hence suffering oversimplification. Some abstract models uses simulated circuit components to emulate the battery behavior [2], hence being tightly connected with specific circuit simulation softwares. Analytical models are abstract models that are derived by simplifying physical models using mathematical analytic methods. Authors of [3] model battery behaviors as a stochastic system based on random walks on a finite state machine. Authors of [10] model the nonlinear relationship between battery capacity and averaged discharge current.

The Rakhmatov-Vrudhula-Wallach (RVW) model proposed in [11] is an abstract model that is derived from solving the diffusion equations governing the electrolytes motion within a battery. The model captures the recovery effect effectively. It has been shown to agree with experimental results and has demonstrated high accuracy in battery capacity prediction and battery life estimation. However, the application of the RVW model requires that the discharge current, as a function of time, is known. This is because the RVW model is typically used at the circuit design stage to evaluate design options on battery discharge. For cyber-physical systems co-design, the approach taken in [6], [7] also assumes known battery discharge profiles for computing tasks. We argue that a dynamic model needs to be established that is able to predict battery capacity based on the discharge current determined by a feedback control law or feedback scheduling algorithm. The magnitude and pulse width of such discharge current can not be determined beforehand.

In this paper, we establish a battery model that captures the recovery effect and supports the co-design of battery management with controller and scheduling algorithms. The model is for the discharge dynamics within one discharge cycle of (possibly rechargeable) batteries. The discharge cycle starts when a battery is fully charged and ends when the battery can not support the demand for discharge current; no recharging is allowed during the cycle. We review the electrochemical battery discharge mechanisms and the RVW model in section 2. In section 3, we derive the dynamic battery model. The optimal impulsive square wave discharge profile is computed in section 4 . In section 5 , we compare battery life for different battery discharge profiles via simulation. Conclusions and future directions are discussed in section 6 .

\section{Battery Mechanism and the RVW model}

This section reviews the relevant properties of electrochemical battery cells and the RVW model. The behaviors of practical batteries are far from an ideal energy source. We summarizes the discussions in [3], [12], [11] and the references therein.

\subsection{The Electrochemical Process}

An electrochemical battery has a cathode and an anode that are connected by electrolyte inside the battery shell. During discharge, the gain of electrons at the cathode (oxidation) is coupled with the loss of electrons at the anode (reduction). The electrochemical reactions, involving electrons $v^{-}$,oxidized species $O$, and reduced species $R$, can be described as follows:

$$
\begin{gathered}
\text { cathode }: O+v^{-} \rightarrow R \\
\text { anode } R-v^{-} \rightarrow O .
\end{gathered}
$$

To simplify the discussion, let us suppose that a battery is symmetric and the two electrodes behave in similar ways. This allows us to consider only species $O$ and the cathode.

Initially, the concentration of species $O$ is uniformly distributed inside a battery. During the discharge period, the species $O$ at the cathode accepts electrons from external circuit and forms species $R$. This causes reduction of $O$ near the cathode, which generates a concentration gradient of $O$ across the battery. Due to this gradient, the species $O$ that is further away from the cathode diffuses to the cathode. The diffusion tends to increase the concentration of $O$ at the cathode. Under constant discharge current, eventually, the diffusion and the consumption reach a balance at the cathode, and the overall concentration of $O$ at the cathode keeps dropping. Once the concentration falls below a certain level, the battery fails to support the outside circuit.

If load is switched off before the battery fails, the cathode fails to draw electrons from the external circuit and thus the transformation at the cathode (from species $O$ to species $R$ ) stops. However, the concentration gradient inside the battery still exists and the diffusion will continue until the concentration gradient disappears. Now species $O$ will become uniformly distributed at a level lower than the initial concentration. The concentration of species $O$ with respect to position is illustrated in Fig. 1.
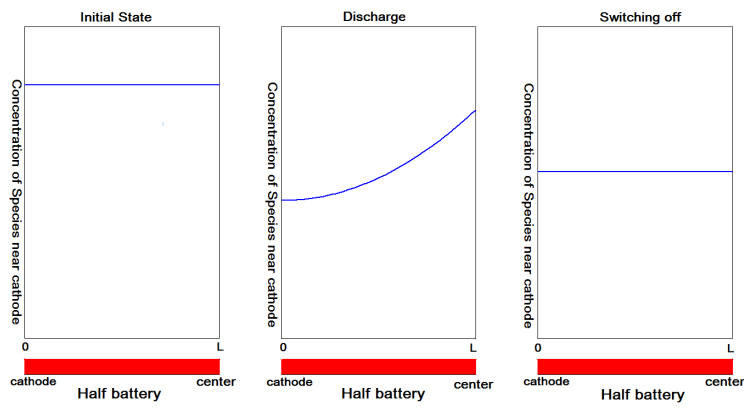

Figure 1. Concentration of species $O$ near the cathode. Left, before discharge; middle, during discharge; right, after discharge.

Batteries behave differently under the impulsive discharge current than under a constant discharge current. When using 
an impulsive discharge current, more species can diffuse to the electrodes when the discharge current drops to zero. Hence, given the same averaged current, the amount of charge delivered under the impulsive current is bigger than that under the constant current.

We define the time period between the initial discharge and the failure of battery as the battery life. Note that the battery life is different from the nominal battery life, which measures battery life under a nominal constant current, and the theoretical battery life, which is equivalent to the total charge contained in the battery.

\subsection{The RVW Model}

Rakhmatov, Vrudhula, and Wallach studied a onedimensional diffusion equation that describes the concentration of electrolytes inside a battery:

$$
\begin{aligned}
J(x, t) & =-D \frac{\partial C(x, t)}{\partial x} \\
\frac{\partial C(x, t)}{\partial t} & =D \frac{\partial^{2} C(x, t)}{\partial x^{2}}
\end{aligned}
$$

where $C(x, t)$ stands for the concentration of electrolyte at time $t$ at the distance $x$ from the electrode, $J(x, t)$ denotes the flux of electrolyte and $D$ is the constant diffusion coefficient. Suppose the length of the battery is $2 w$. Then the boundary condition for (1) can be derived as,

1) $x=0$ : according to Faraday's law, the flux of electrolyte $J(0, t)$ at the electrode is proportional to the current $i(t)$ supplied by the battery.

$$
-J(0, t) \nu A F=i(t)
$$

where $F$ stands for the Faraday Constant, $A$ stands for the cross section of the electrode, and $\nu$ is a scaling factor. One can derive the first boundary condition as

$$
\left.D \frac{\partial C(x, t)}{\partial x}\right|_{x=0}=\frac{i(t)}{\nu F A} .
$$

2) $x=w$ : the concentration gradient at the center of the battery is zero. Thus, one can derive the second boundary condition as

$$
\left.D \frac{\partial C(x, t)}{\partial x}\right|_{x=w}=0
$$

To solve (1), the Laplace transform method is applied. Since the electrolyte is uniformly distributed when discharge started, $C(x, 0)$ is a constant and can be denoted by $C^{\star}$. The solution for the concentration of electrolyte at the electrode is

$$
C(0, t)=C^{\star}-\frac{i(t)}{\nu w F A} *\left(1+2 \sum_{m=1}^{\infty} e^{-\frac{\pi^{2} m^{2}}{w^{2}} D t}\right)
$$

where $\star$ stands for the convolution. Define $\rho(t)$ as

$$
\begin{aligned}
\rho(t) & =1-\frac{C(0, t)}{C^{\star}} \\
& =\frac{i(t)}{C^{\star} \nu w F A} *\left(1+2 \sum_{m=1}^{\infty} e^{-\frac{\pi^{2} m^{2}}{w^{2}} D t}\right)
\end{aligned}
$$

At the starting time $t=0, C(0,0)=C^{\star}$ and $\rho(0)=0$. As the discharge continues, $C(0, t)$ gradually decreases and $\rho(t)$ increases. Finally at the dead time $t=L, C(0, L)$ drops to the cutoff level $C_{\text {cutoff }}$ while $\rho(t)$ reaches the corresponding threshold $\rho^{\star}=\left(1-\frac{C_{\text {cutoff }}}{C^{\star}}\right)$. Note that both $\rho^{\star}$ and $C_{\text {cutoff }}$ are constants associate with a given battery.

\section{A Dynamic Discharge Model}

The RVW model relates the concentration at the electrode with the discharge current $i(t)$. But the relationship is static in the sense that the current $i(t)$ must be known for the entire discharge cycle. We present a state space dynamic model that produces the same input-output relationship as the RVW model. This state space model is linear and time invariant, hence easier to use than the RVW model.

Let $\lambda_{\mathrm{m}}=\frac{\pi^{2} D}{w^{2}} m^{2}$ and $\alpha=C^{\star} \nu w F A \rho^{\star}$. To normalize $\rho(t)$, we define $y(t)$ as

$$
y(t)=\frac{\rho(t)}{\rho^{\star}}=\frac{i(t)}{\alpha} *\left(1+2 \sum_{\mathrm{m}=1}^{\infty} e^{-\lambda_{\mathrm{m}} t}\right)
$$

which implies that $y(0)=0$ at the starting time $t=0$ and $y(L)=1$ at the dead time $t=L$.

To derive the state space model, we first replace $m=\infty$ with $m=M$ in (6) and obtain

$$
\begin{aligned}
y(t) & =\frac{i(t)}{\alpha} * 1+\frac{i(t)}{\alpha} * 2 \sum_{\mathrm{m}=1}^{\mathrm{M}} e^{-\lambda_{m} t} \\
& =\left[\begin{array}{llll}
1 & 1 & \cdots & 1
\end{array}\right]\left[\begin{array}{c}
\frac{i(t)}{\alpha} * 1 \\
\frac{2 i(t)}{\alpha} * e^{-\lambda_{1} t} \\
\cdots \\
\frac{2 i(t)}{\alpha} * e^{-\lambda_{\mathrm{M}} t}
\end{array}\right]
\end{aligned}
$$

Next, we define the state variables to be $x=$ $\left[x_{0}(t), \ldots, x_{\mathrm{M}}(t)\right]^{T}$. The elements of $x$ satisfy,

$$
\begin{cases}\dot{x}_{0}(t)=\frac{1}{\alpha} i(t) & \\ \dot{x}_{\mathrm{m}}(t)=-\lambda_{\mathrm{m}} x_{\mathrm{m}}(t)+\frac{2}{\alpha} i(t) & \mathrm{m} \in\{1,2, \cdots, \mathrm{M}\} \\ x_{\mathrm{m}}(0)=0 & \mathrm{~m} \in\{0,1, \cdots, \mathrm{M}\}\end{cases}
$$

The solutions of the above equations are,

$$
\left\{\begin{aligned}
x_{0}(t) & =\int_{0}^{t} \frac{1}{\alpha} i(\tau) d \tau=\frac{i(t)}{\alpha} * 1 \\
x_{\mathrm{m}}(t) & =\int_{0}^{t} e^{-\lambda_{\mathrm{m}}(t-\tau)} \frac{2}{\alpha} i(\tau) d \tau \\
& =\frac{2 i(t)}{\alpha} * e^{-\lambda_{\mathrm{m}} t}
\end{aligned}\right.
$$


We substitute (8) into (7) to get,

$$
y(t)=[1,1, \ldots, 1]\left[\begin{array}{c}
x_{0}(t) \\
x_{1}(t) \\
\ldots \\
x_{\mathrm{M}}(t)
\end{array}\right]=[1,1, \ldots, 1] x(t)
$$

To this point, we have established the dynamic battery discharge model as

$$
\begin{aligned}
& \dot{x}(t)=A x(t)+b i(t) \\
& y(t)=c x(t)
\end{aligned}
$$

where

$$
\begin{aligned}
& A=\left[\begin{array}{llll}
0 & & & \\
& -\lambda_{1} & & \\
& & \ldots & \\
& & & -\lambda_{\mathrm{M}}
\end{array}\right]_{(\mathrm{M}+1) \times(\mathrm{M}+1)} \\
& b=\left[\begin{array}{c}
\frac{1}{\alpha} \\
\frac{2}{\alpha} \\
\cdots \\
\frac{2}{\alpha}
\end{array}\right]_{(\mathrm{M}+1) \times 1} c=[1,1, \cdots, 1]_{1 \times(\mathrm{M}+1)} .
\end{aligned}
$$

In addition, we have shown that this model is input-output equivalent to the RVW model when $M \rightarrow \infty$.

According to (10), we know that $y(t)$ is the sum of all internal states $x_{\mathrm{i}}(t)$. We now discuss the role played by the internal states. To simplify the analysis, we begin with only two internal states and a simplified dynamic model as

$$
\left\{\begin{aligned}
{\left[\begin{array}{c}
\dot{x}_{0}(t) \\
\dot{x}_{1}(t)
\end{array}\right] } & =\left[\begin{array}{cc}
0 & 0 \\
0 & -\lambda_{1}
\end{array}\right]\left[\begin{array}{l}
x_{0}(t) \\
x_{1}(t)
\end{array}\right]+\left[\begin{array}{c}
\frac{1}{\alpha} \\
\frac{2}{\alpha}
\end{array}\right] i(t) \\
y(t) & =x_{0}(t)+x_{1}(t)
\end{aligned}\right.
$$

Suppose a constant discharge current is drawn. We select the physical parameters of a battery to be

$$
I=222.7 m A \quad \alpha=40375 \quad \lambda_{m}=(0.2)^{2} m^{2} .
$$

The system output $y(t)$, internal states $x_{0}(t)$ and $x_{1}(t)$ are plotted in Fig. 2. It shows that the $x_{0}(t)$ is a straight line. The $x_{1}(t)$ is transient from $t=0$ to $t=S$ and then approaches the constant steady state from $t=S$ to $t=L$. The constant $x_{1}(t)_{\text {steady }}$ represents the wasted electrolytes that can not be drawn by the current. Thus to maximize battery efficiency, we need to minimize $x_{1}(t)_{\text {steady }}$.

\section{Impulsive Discharge Current}

The co-design for cyber-physical systems should take advantage of the recovery effect to improve the battery efficiency. To achieve this goal, we are interested in studying different discharge profiles. In this paper, we focus on the periodic impulsive current $i(t)$ shown in Fig. 3 .

The periodic square wave can be expressed as

$$
\begin{cases}i(t)=u & t_{1} \leq t \leq t_{1}+Q \\ i(t)=0 & t_{1}+Q \leq t \leq t_{1}+T\end{cases}
$$

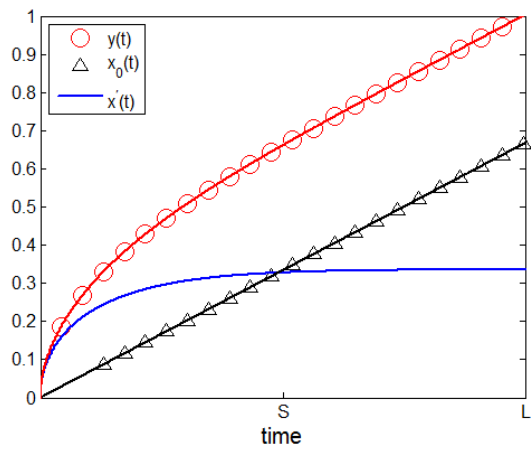

Figure 2. Internal States

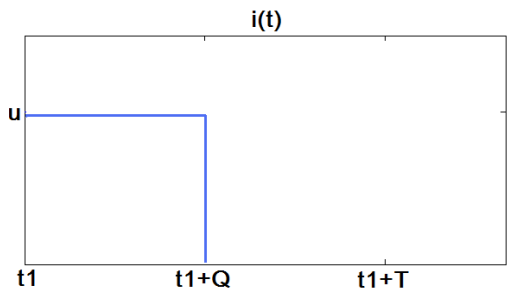

Figure 3. Impulse Current

where $u$ is a constant. From Fig. 3, it can be seen that the shape of $i(t)$ depends on two parameters: the duty ratio $R=$ $\frac{Q}{T}$ and the length of the period $T$.

We now derive the optimal square wave discharge profile under a fixed average current $i_{\text {av }}$, i.e.

$$
u \cdot \frac{Q}{T}=i_{\mathrm{av}} \text {. }
$$

We first determine the optimal duty $R$ in the transient given that $T$ is fixed. Then we determine the optimal period $T$ when the system is near steady state given the optimal duty $R$.

To measure the increase of the state $x_{1}$ in transient, we define $f$ to be the difference between $x_{1}\left(t_{0}+T\right)$ and $x_{1}\left(t_{0}\right)$ as a function of $R$

$$
\begin{aligned}
f(R) & =x_{1}\left(t_{0}+T\right)-x_{1}\left(t_{0}\right) \\
& =e^{-\lambda_{1} T} x\left(t_{0}\right)-x_{1}\left(t_{0}\right) \\
& +\frac{2}{\alpha} \frac{i_{\mathrm{av}}}{R} \frac{1}{\lambda_{1}}\left(e^{-\lambda_{1}(T-R T)}-e^{-\lambda_{1} T}\right) .
\end{aligned}
$$

We want to show that $f(R)$ is monotonic increasing with respect to $R>0$. Hence the optimal duty cycle is the minimum value for $R$.

To justify this conclusion, we compute the derivative of $f(R)$ with respect to $R$ as

$$
\begin{gathered}
\frac{d f}{d R}=\frac{2}{\alpha}\left(-\frac{i_{\mathrm{av}}}{R^{2}}\right) \frac{1}{\lambda_{1}}\left(e^{-\lambda_{1}(T-R T)}-e^{-\lambda_{1} T}\right) \\
\quad+\frac{2}{\alpha} \frac{i_{\mathrm{av}}}{R} T e^{-\lambda_{1}(T-R T)} \\
=\frac{2}{\alpha} \frac{i_{\mathrm{av}}}{R^{2}} e^{-\lambda_{1} T}\left(-\frac{1}{\lambda_{1}}\left(e^{\lambda_{1} R T}-1\right)+R T e^{\lambda_{1} R T}\right) .
\end{gathered}
$$


Define $H(R)$ to be

$$
H(R)=\left(-\frac{1}{\lambda_{1}}\left(e^{\lambda_{1} R T}-1\right)+R T e^{\lambda_{1} R T}\right) .
$$

Then,

$$
\left\{\begin{array}{l}
H(0)=0 \\
\frac{d H}{d R}=R T^{2} \lambda_{1} e^{\lambda_{1} R T}
\end{array}\right.
$$

that implies $\frac{d H}{d R}>0$ for all $R>0$, i.e. $H(R)$ is monotone increasing. Since $H(0)=0$, we can conclude that $H(R)>$ 0 if $R>0$. Moreover, plug (15) back into (14),

$$
\frac{d f}{d R}=\frac{2}{\alpha} \frac{i_{\mathrm{av}}}{R^{2}} e^{-\lambda_{1} T} H(R)
$$

we must have that

$$
\frac{d f}{d R}>0 \quad \text { if } \mathrm{R}>0,
$$

which means that the function $f$ is monotone increasing whenever $R>0$. Therefore, the shorter the duty ratio, the better battery efficiency is.

Suppose $R_{L}$ is the minimum allowed duty. Next, we determine the optimal $T$ given $R=R_{\mathrm{L}}$ fixed for the steady state. From Fig. 2, we can see that the steady state value of $x_{1}(t)$, denoted by $x_{1}(t)_{\text {steady }}$, stands for the total unavailable capacity that needs to be minimized. The conclusion is that $x_{1}(t)_{\text {steady }}$ is monotone decreasing with respect to $T$. Hence the longer the discharge period is, the more efficient the battery is.

The justification to the conclusion is as follows. According to the property of the steady state, we have

$$
x_{1}\left(t_{0}\right)_{\text {steady }}=x_{1}\left(t_{0}+T\right)_{\text {steady }} .
$$

Moreover,

$$
\begin{aligned}
& x_{1}\left(t_{0}+T\right)_{\text {steady }} \\
& =e^{-\lambda_{1} T} x_{1}\left(t_{0}\right)_{\text {steady }}+\frac{2 i_{\text {av }} T}{\lambda_{1} \alpha Q}\left(e^{-\lambda_{1}(T-Q)}-e^{-\lambda_{1} T}\right)
\end{aligned}
$$

Therefore,

$$
x_{1}\left(t_{0}\right)_{\text {steady }}=\frac{2 i_{\mathrm{av}} T}{\lambda_{1} \alpha Q} \frac{e^{-\lambda_{1}(T-Q)}-e^{-\lambda_{1} T}}{1-e^{-\lambda_{1} T}}
$$

Since the ratio of a duty cycle has been fixed as $R_{\mathrm{L}}$, we have,

$$
Q=R_{\mathrm{L}} T
$$

Substitute (18) into (17), we have,

$$
x_{1}\left(t_{0}\right)_{\text {steady }}=\frac{2 i_{\mathrm{av}}}{\lambda_{1} \alpha R_{\mathrm{L}}} \frac{e^{\lambda_{1} R_{\mathrm{L}} T}-1}{e^{\lambda_{1} T}-1}
$$

The state $x_{1}\left(t_{0}\right)_{\text {steady }}$ can now be viewed as a function of $T$. Using similar methods as the discussion for the optimal duty ratio $R$. We can show that $\frac{d x_{1}\left(t_{0}\right)_{\text {steady }}}{d T}<0$. Therefore $x_{1}\left(t_{0}\right)_{\text {steady }}$ is monotone decreasing. The longer the period $T$, the smaller $x_{1}\left(t_{0}\right)_{\text {steady }}$ is, and the better the battery efficiency is.

\section{Simulation Results}

We now compare simulation results for a battery with the following parameters:

$$
I=222.7 m A \quad \alpha=40375 \quad \lambda_{m}=(0.2)^{2} m^{2}
$$

under different discharge profiles.

First, the influence of duty ratio is shown in Fig. 4. The time period is fixed to be $T=L / 300$ where $L$ is the battery life. We can see that the smaller the duty ratio $R$, the smaller $y(t)$ is, i.e. the more efficient the battery is, which is consistent with our analysis.

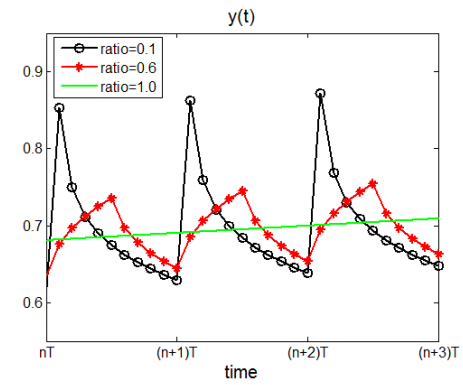

Figure 4. Battery capacity under different duty ratios

The influence of the time period $T$ is shown in Fig. 5. The duty ratio is fixed to be $R=0.5$. The right picture indicates that the larger the time period $T$, the smaller $x_{1}(t)$ is, i.e. the less unavailable species in the battery. The left picture shows that the larger the time period $T$, the smaller $y(t)$ is, i.e. the more efficient the battery is. This is consistent with our analysis.
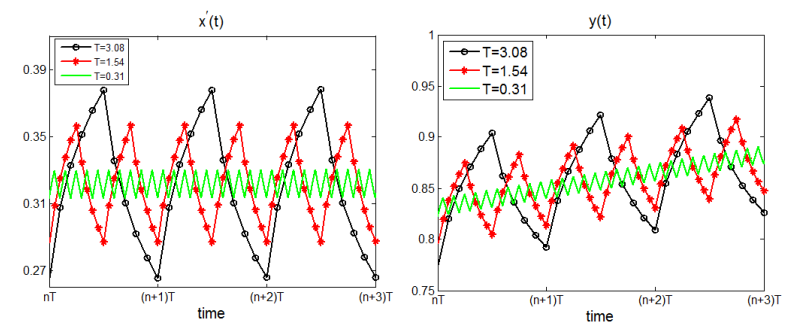

Figure 5. Battery capacity under different discharge periods.

It can also be observed that as the discharge current becomes more impulsive i.e. the ratio becomes smaller or the period becomes larger, although the unused capacity at the beginning of each discharge period decreases, the maximal unused capacity during the discharge period increases. This will cause problems when the battery is close to failure since the high unused capacity fails the battery before the recovery effect takes place. Therefore, if the discharge current is adjustable, an optimal discharge strategy should be more impulsive at the beginning of the discharge and gradually 
become less impulsive till the end. This agrees with the existing simulation and experimental results in the literature reviewed.

\section{Conclusions}

We introduce a dynamic battery discharge model that is input-output equivalent to the RVW model. Since the accuracy of RVW model has been verified by experimental data, it is reasonable to conclude that the dynamic battery discharge model inherits such accuracy. The states of this model represent the instantaneous discharge/recovery effects within the battery. Using this dynamic model, the battery can be viewed as a dynamic system under the control $i(t)$. Optimal $i(t)$ can be determined analytically without seeking numerical methods.

The dynamic battery model opens doors to many research questions for cyber-physical systems. Based on the simple case for square wave discharge current, a natural extension is to design pulse width modulation (PWM) schemes to generate discharge current profile when $i_{\mathrm{av}}$ is a function of time that depends on feedback control and feedback scheduling. Note that although we assume that $i(t)=0$ after the impulse in this paper, the results still hold when a background discharge current is imposed. The background discharge current usually exists in real systems, for example, to keep the battery management circuits alive. Of course, a design question will be whether the extra cost in the background discharge supersedes the gain for implementing battery management algorithms. Suppose the savings are significant, then power electronic circuits will make such PWM schemes implementable.

\section{Acknowledgments}

We would like to thank Dr. Xue Liu for discussion and an invitation to submit this paper. The research is supported by ONR grant N00014-08-1-1007, NSF grant 0841195, and NSF grant 0720536.

\section{References}

[1] T. Chantem, X. S. Hu, and M. Lemmon, "Generalized elastic scheduling," in Proc. 27th RTSS, 2006.

[2] M. Chen and G. A. Rincon-Mora, "Accurate electrical battery model capable of predicting runtime and iv performance," IEEE transactions on energy conversion, vol. 21, pp. 504511,2006

[3] C.-F. Chiasserini and R. Rao, "Energy efficient battery management," IEEE Journal on Selected Areas in Communications, vol. 19, no. 7, pp. 1235-1245, 2001.

[4] J. Cortés, S. Martínez, T. Karatas, and F. Bullo, "Coverage control for mobile sensing networks," IEEE Transactions on Robotics and Automation, vol. 20, no. 2, pp. 243-255, 2004.
[5] M. Doyle, T. Fuller, and J. Newman, "Modeling of galvanostatic charge and discharge of the lithium/polymer/insertion cell," J. Electrochemical Soc., vol. 140, no. 6, pp. 1526-1533, 1995.

[6] L. Jiong and N. K. Jha, "Power-efficient scheduling for heterogeneous distributed real-time embedded systems," IEEE Transactions on Computer-Aided Design of Integrated Circuits and Systems, vol. 26, no. 6, pp. 1161-1170, 2007.

[7] L. Jiong, N. K. Jha, and L.-S. Pei, "Simultaneous dynamic voltage scaling of processors and communication links in realtime distributed embedded systems," IEEE Transactions on Very Large Scale Integration (VLSI) Systems, vol. 15, no. 4, pp. 427-437, 2007.

[8] D. Linden and T. Reddy, Handbook of Batteries, 3rd ed. McGraw-Hill, 2001.

[9] X. Liu, Q. Wang, W. He, M. Caccamo, and L. Sha, "Optimal real-time sampling rate assignment for wireless sensor networks," ACM Transactions on Sensor Networks (TOSN), vol. 2, no. 2, pp. 263-295, 2006.

[10] M. Pedram and Q. Wu, "Battery-powered digital CMOS design," IEEE Transactions on VLSI Systems, vol. 10, no. 5, pp. 601-607, 2002.

[11] D. Rakhmatov, S. Vrudhula, and D. A. Wallach, "A model for battery lifetime analysis for organizing applications on a pocket computer,' IEEE Transactions on VLSI Systems, vol. 11, no. 6, pp. 1019-1030, 2003.

[12] R. Rao, S. Vrudhula, and D. N. Rakhmatov, "Battery modeling for energy-aware system design," Computer, vol. 36, no. 12, pp. 77-87, 2003.

[13] L. Sha, T. Abdelzaher, K.-E. Årzén, A. Cervin, T. Baker, A. Burns, G. Buttazzo, M. Caccamo, J. Lehoczky, and A. K. Mok, "Real time scheduling theory: A historical perspective," Real-Time Systems, vol. 28, no. 2-3, pp. 101-155, 1994.

[14] F. Xia and Y. Sun, "Control-scheduling codesign: A perspective on integrating control and computing," in Dynamics of Continuous, Discrete and Impulsive Systems - Series B: Applications and Algorithms, Special Issue on ICSCA'06. Rome, Italy: Watam Press, 2006, pp. 1352-1358.

[15] F. Zhang, D. M. Fratantoni, D. Paley, J. Lund, and N. E. Leonard, "Control of coordinated patterns for ocean sampling," International Journal of Control, vol. 80, no. 7, pp. 1186-1199, 2007.

[16] F. Zhang and N. E. Leonard, "Coordinated patterns of unit speed particles on a closed curve," Systems and Control Letters, vol. 56, no. 6, pp. 397-407, 2007.

[17] F. Zhang, K. Szwaykowska, V. Mooney, and W. Wolf, "Task scheduling for control oriented requirements for cyberphysical systems," in Proc. of 29th IEEE Real-Time Systems Symposium (RTSS 2008), Barcelona, Spain, 2008, pp. 47-56. 